\title{
El niño con estridor persistente
}

\section{The child with persistent stridor}

\author{
Andrés Alvo ${ }^{\mathrm{a}}$ \\ aOtorrinolaringólogo, Clínica Alemana de Santiago. Hospital de Niños Roberto del Río. Hospital Clínico Universidad de Chile. Santiago, Chile
}

Recibido: 17 de marzo de 2020; Aceptado: 4 de julio de 2020

¿Qué se sabe del tema que trata este estudio?

Existen distintas patologías de la vía aérea laringotraqueal pediátrica que cursan con estridor y dificultad respiratoria, que determinan una estrechez luminal o bien un colapso dinámico de sus estructuras. De la indicación clásica de traqueostomía y de grandes cirugías abiertas se ha ido pasando a técnicas endoscópicas menos invasivas.
¿Qué aporta este estudio a lo ya conocido?

En los últimos años, han aparecido nuevos conceptos respecto al manejo del estridor pediátrico. Esta revisión no sólo describe las técnicas más actuales, si no que profundiza en diagnósticos menos conocidos, resumiendo el estado del arte en el diagnóstico y manejo del estridor pediátrico persistente.

\section{Resumen}

El estridor es un ruido respiratorio anormal generado por obstrucción o colapso de la vía aérea laringotraqueal, de manera aguda o de evolución crónica. Existen distintas causas tanto congénitas como adquiridas capaces de producir dificultad respiratoria, que puede llegar a ser grave y con potencial riesgo vital. El diagnóstico clínico del paciente con estridor persistente debe ser complementado con un estudio endoscópico de la vía aérea y en ocasiones con imágenes, para intentar determinar el o los sitios comprometidos y posibles malformaciones asociadas. La indicación de tratamiento debe ser individualizada, considerando el estado general del paciente, las etiologías responsables, el impacto sobre la respiración y la deglución, el pronóstico y la capacidad técnica del equipo tratante, entre otras. Las alternativas pueden incluir observación, medidas no farmacológicas, medicamentos locales o sistémicos, procedimientos endoscópicos, cirugías abiertas, o bien la instalación de una traqueostomía de manera temporal o como manejo definitivo. El objetivo de esta revisión es entregar un adecuado conocimiento de la fisiopatología y la etiopatogenia del estridor pediátrico persistente, fundamental para el correcto manejo de estos pacientes complejos, que debiera realizarse idealmente en un contexto multidisciplinario.
Palabras clave:

Estridor;

Vía Aérea Pediátrica;

Laringomalacia;

Parálisis Cordal;

Estenosis Subglótica

Correspondencia:

Andrés Alvo

andresalvo@gmail.com 


\begin{abstract}
Stridor is an abnormal respiratory sound caused by obstruction or collapse of the laryngotracheal airway, either acutely or chronically. There are different causes, both congenital and acquired, that can produce shortness of breath which may be severe and potentially life-threatening. The clinical diagnosis must be complemented with an endoscopic airway assessment and sometimes with imaging, to try to determine the areas involved and possible associated malformations. Treatment should be individualized, considering the patient's overall condition, stridor etiology, its impact on breathing and swallowing, prognosis, and technical capacity of the managing team, among others. Alternatives may include observation, non-pharmacological measures, local or systemic medications, endoscopic and open surgeries, or a temporary or long-term tracheostomy. A thorough understanding of the pathophysiology and etiopathogenesis of persistent pediatric stridor is essential for the correct management of these complex patients, ideally in a multidisciplinary manner.
\end{abstract}

\section{Keywords:}

Stridor;

Pediatric Airway;

Laryngomalacia;

Vocal Cord Paralysis;

Subglottic Stenosis

\section{Introducción}

Enfrentarse a un niño con estridor y signos de dificultad respiratoria puede ser una experiencia estresante para el equipo médico. La gran variedad de posibles diagnósticos diferenciales y la necesidad de mantener una vía aérea estable y permeable plantean escenarios clínicos complejos.

Podemos definir estridor como un ruido respiratorio anormal debido al flujo de aire a través de una vía aérea estrecha o colapsada, que se origina en la laringe, tráquea y/o bronquios principales. Sus causas pueden ser tanto agudas como progresivas o crónicas, y deberse a condiciones congénitas o adquiridas.

Según la localización y característica de la obstrucción, el sonido puede ser de distintos tipos y ubicarse en cualquier momento del ciclo respiratorio. Colapsos dinámicos de la vía aérea extratorácica (habitualmente supraglóticos) generan un estridor inspiratorio, mientras que los colapsos dinámicos de la vía aérea intratorácica (tráquea distal y bronquios) se manifiestan con estridor espiratorio. Por otro lado, las obstrucciones fijas de la vía aérea laringotraqueal (habitualmente subglóticas o glóticas) se manifiestan por estridor bifásico ${ }^{1}$.

Existen también otros ruidos respiratorios patológicos que deben ser diferenciados del estridor pues se originan en otras estructuras de la vía aérea. Así, se describe el estertor nasal como el sonido generado por congestión a nivel de las fosas nasales; el ronquido como un ruido áspero y grave ocasionado por vibración de las estructuras velares y faríngeas; y las sibilancias como un sonido agudo y musical, habitualmente al final de la espiración, originado en la vía aérea inferior distal ${ }^{2}$. Diferenciar entre uno y otro tipo de respiración ruidosa no siempre es fácil. Más aún, pueden coexistir más de un tipo en un mismo paciente, ser intermitentes o modificarse con la actividad o posición, o bien mezclarse con otros ruidos como secreciones o crepitaciones.
Si bien el estridor es habitualmente la causa que motiva el estudio del paciente, son los síntomas y signos acompañantes los que determinarán la gravedad del problema. En general, signos de esfuerzo respiratorio excesivo como retracciones torácicas, la presencia de cianosis o desaturaciones, el mal incremento ponderal u otros trastornos de la alimentación y deglución, comandarán la decisión de observar o de realizar alguna intervención. Estos fenómenos pueden variar en el tiempo, ir progresando a medida que el niño va haciéndose más activo o la patología empeora, o deteriorarse bruscamente al sobreagregarse una infección aguda de la vía aérea.

Hasta hace algunas décadas, la mayoría de estos pacientes eran sometidos a una traqueostomía como método de bypass de la zona anatómica comprometida, con los riesgos y dificultades que ésta representa. En el último tiempo han aparecido numerosos avances que han mejorado el diagnóstico y tratamiento de las patologías que cursan con estridor y dificultad respiratoria, permitiendo corregir de manera más dirigida el trastorno subyacente.

El objetivo de esta revisión es entregar un adecuado conocimiento de la fisiopatología y la etiopatogenia del estridor pediátrico persistente, fundamental para el correcto manejo de estos pacientes complejos, que debiera realizarse idealmente en un contexto multidisciplinario. A continuación, revisaremos el enfoque diagnóstico y terapéutico general del paciente pediátrico con estridor persistente, las causas más comunes y los aspectos específicos más relevantes de cada patología.

\section{Estudio del paciente con estridor}

La evaluación complementaria más importante en el paciente con estridor es el estudio endoscópico. La nasofaringolaringoscopía flexible (o nasofibroscopía) en el paciente despierto permite una evaluación di- 
námica de la vía aérea desde la nariz hasta las cuerdas vocales. Este examen puede también realizarse en pacientes bajo sueño inducido por drogas (drug-induced sleep endoscopy o DISE).

Si los síntomas son severos o se sospecha una alteración bajo el nivel de las cuerdas vocales, la revisión de la vía aérea se continua con una laringotraqueobroncoscopía (LTB), que puede ser realizada con instrumentos rígidos y/o flexibles ${ }^{3}$. Ambas poseen ventajas y desventajas, por lo que son complementarias.

Los estudios por imágenes tienen un papel más secundario en estos pacientes. Están indicados ante sospecha de patologías asociadas; compresiones extrínsecas, tumores o malformaciones vasculares; cuerpos extraños; o ante vías aéreas muy estrechas difícilmente evaluables mediante endoscopía. La tomografía computarizada (TC) es el estudio por imágenes de elección en la mayoría de los casos, con o sin estudio angiográfico. En los últimos años, también se ha descrito el uso de la llamada "broncoscopía virtual" mediante $\mathrm{TC}^{4,5}$. Otros exámenes pueden incluir radiografía simple, ecotomografía, resonancia magnética y estudios contrastados como broncografía o videofluoroscopía de deglución.

Los estudios dirigidos a buscar comorbilidades (por ejemplo, endoscopía digestiva alta o pHmetría) o consecuencias de la obstrucción respiratoria (por ejemplo, saturometría nocturna o polisomnografía) serán indicadas según la sospecha clínica y el estado de cada paciente.

\section{Aspectos generales del manejo del paciente con estridor}

Aunque el tratamiento específico dependerá de las condiciones del paciente, el impacto del trastorno sobre su sistema cardio-respiratorio y de las patologías responsables, podemos describir algunas ideas generales respecto al manejo. En primer lugar, estos pacientes debiesen ser discutidos idealmente en un comité aerodigestivo multidisciplinario, en especial los casos más complejos ${ }^{6}$.

En casos leves, en los que no hay signos de alarma, muchas veces se puede tomar una conducta expectante, especialmente en trastornos no progresivos que tienden a resolución espontánea.

Algunos pacientes pueden beneficiarse de medidas generales tales como posicionar de lado y semisentado para aliviar el colapso de la base lingual; tratar la inflamación y el reflujo gastroesofágico; rehabilitación de la deglución o vías de alimentación alternativa; y/o uso de cánulas nasales de alto flujo o ventilación mecánica no invasiva.

En casos más severos, en los que se opte por un manejo quirúrgico, se debe contar con una evaluación completa de la vía aérea y abordar los niveles con compromiso significativo. En lo posible se deben elegir procedimientos menos invasivos o potencialmente reversibles, en especial si la patología puede tender a la resolución espontánea. $\mathrm{Si}$, por el contrario, la patología es grave y persistente, a veces se requerirá de cirugías extensas y complejas. Debe considerarse que la mejoría no siempre será completa, que pueden requerirse múltiples intervenciones en el tiempo y que las expectativas deben ajustarse a cada caso particular.

En otras ocasiones, la mejor opción -temporal o definitiva- puede ser una traqueostomía. Entre sus potenciales ventajas se incluye evitar el daño quirúrgico a la estructura laríngea, saltarse o dar soporte a la zona alterada, mejorar la tolerancia a la ventilación mecánica, facilitar el manejo de secreciones, entre otras. Las desventajas incluyen el cuidado especializado de la traqueostomía, necesidad de entrenamiento de los cuidadores y sus potenciales complicaciones tempranas y $\operatorname{tardías}^{7,8}$.

\section{Principales causas de estridor pediátrico persistente}

El estridor en niños puede ser congénito, adquirido o mixto. Una o más causas pueden coexistir en el mismo paciente y uno o más niveles de la vía aérea pueden estar comprometidos. Algunos diagnósticos, como la parálisis cordal, pueden ser tanto congénitos como adquiridos, y otros, como la traqueomalacia, pueden deberse tanto a un defecto primario como a una causa secundaria.

\section{Laringomalacia}

Es la causa más frecuente de estridor congénito, representando al menos un $60 \%$ de los casos. Los síntomas suelen manifestarse entre las 2 a 4 semanas de vida, cuando el recién nacido comienza a tener más actividad. Su causa no es del todo clara, aunque se piensa que se debería a inmadurez neuromuscular, más que a una alteración cartilaginosa. El estridor habitualmente aumenta hasta los 6 meses de edad, para disminuir y desaparecer hacia los 12-18 meses $^{9}$. Los casos secundarios a patologías neurológicas o secuelas quirúrgicas también existen, pero son mucho más infrecuentes. ${ }^{10}$

Se presenta con estridor inspiratorio debido al colapso dinámico de estructuras supraglóticas, clasificadas por Olney en: tipo I, colapso de mucosa aritenoidea; tipo II, repliegues ariepiglóticos cortos; tipo III, colapso epiglótico en retroflexión ${ }^{11}$.

El trastorno es leve en el 90\% de los casos, mientras que en el $10 \%$ restante se asocia a cianosis, retracciones, dificultad en la alimentación o retraso 
pondoestatural, considerándose moderado-severo, ${ }^{9,12}$. Puede asociarse a una segunda alteración de la vía aérea en un porcentaje variable $(7,5 \text { a } 64 \%)^{13}$, lo que refuerza la necesidad de completar el estudio endoscópico de la vía aérea cuando se plantea un manejo quirúrgico.

Casos leves pueden ser seguidos de manera expectante, aunque también se pueden recomendar medidas posturales y el uso de inhibidores de la bomba de protones ${ }^{14}$. Casos moderados-severos tienen indicación quirúrgica endoscópica. La supraglotoplastía consiste en seccionar y/o resecar tejido con la finalidad de disminuir la malacia (figura 1). En casos de colapso epiglótico se indica una epiglotopexia, en la que ésta se fija a la base de la lengua ${ }^{15}$.

\section{Inmovilidad cordal}

La disminución de la movilidad de una o ambas cuerdas vocales se detectará habitualmente en la nasofibroscopía. Esta puede deberse a un trastorno neural (paresia y parálisis) o a restricción mecánica (por cicatrices, luxación o fijación cricoaritenoidea ${ }^{16}$. Para diferenciar entre ambas, los aritenoides y las cuerdas deben ser palpadas durante una laringoscopía bajo anestesia. En casos de parálisis idiopática se aconseja también obtener una resonancia magnética cerebral.

\section{a) Paresia y parálisis cordal}

Salvo excepciones, las parálisis cordales que cursan con estridor y dificultad respiratoria son las bilaterales en posición cerrada, mientras que las unilaterales se caracterizan más bien por disfonía y ocasionalmente por trastornos de la deglución.

Son la segunda causa más frecuente de estridor congénito, pudiendo ser idiopáticas o bien secundarias a malformaciones neurológicas (ej: Arnold-Chiari),

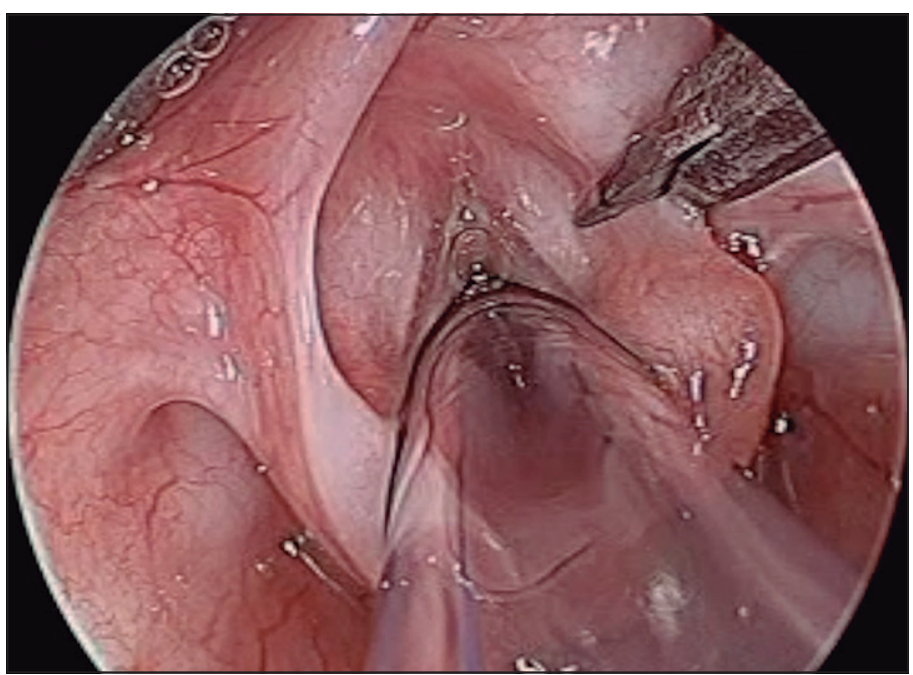

Figura 1. Sección de repliegue ariepiglótico durante una supraglotoplastía. elongación neural durante parto, u otras. Por otra parte, las causas adquiridas son generalmente iatrogénicas, después de cirugía cervical o cardiotorácica ${ }^{17}$.

Alrededor del 50\% de los casos podrían resolverse espontáneamente en los primeros 6-12 meses, en especial en casos idiopáticos, asociados a trauma del parto, o por manipulación quirúrgica sin lesión directa ${ }^{18}$.

El manejo dependerá de la condición del paciente y el pronóstico de la lesión. Algunos casos pueden manejarse de manera expectante o con ventilación mecánica no invasiva. Aún así, más del 50\% de las parálisis cordales bilaterales en posición cerrada requerirán tratamiento quirúrgico. La traqueostomía ha sido históricamente el gold standard, pero se asocia a los problemas antes descritos ${ }^{19}$. En el último tiempo se han desarrollado técnicas poco invasivas orientadas a aumentar el lumen glótico (figura 2), entre las que se encuentran la lateralización cordal percutánea ${ }^{20}$, el split cricoideo endoscópico ${ }^{21,22}$ y la inyección de toxina botulínica en los músculos cricotiroideos ${ }^{23,24}$. Técnicas más agresivas como la aritenoidectomía parcial y la cordotomía posterior ${ }^{19}$ debieran reservarse para casos irreversibles en pacientes mayores, en los que otras técnicas han fallado.

\section{b) Inmovilidad de origen mecánico}

Condiciones como estenosis glótica posterior o subluxación cricoaritenoidea pueden presentarse de manera similar, en especial si el compromiso es bilateral. El diagnóstico se realiza mediante palpación durante la laringoscopía y el tratamiento puede incluir reducción endoscópica, sección de cicatrices, infiltración de

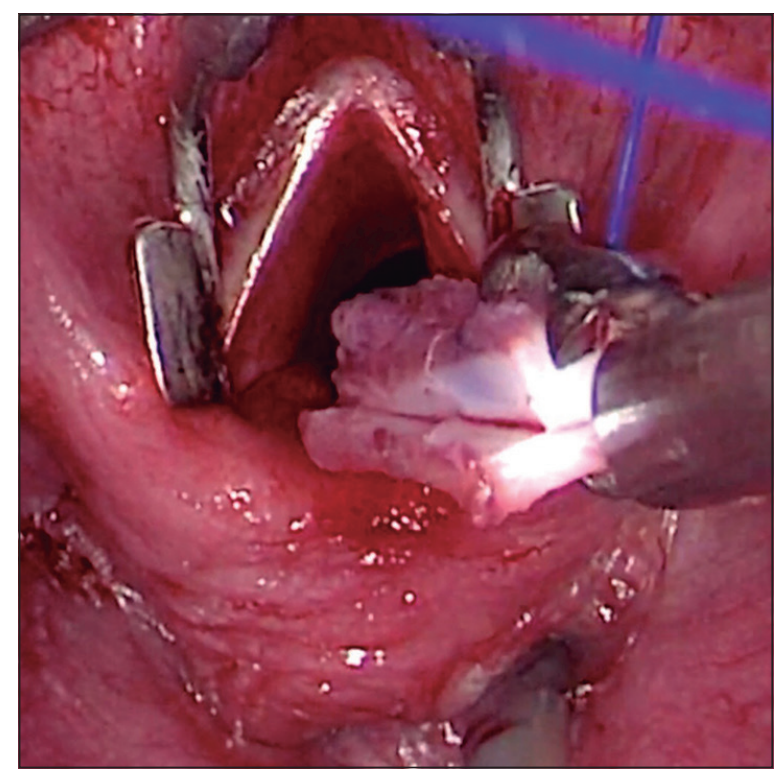

Figura 2. División cricoidea posterior endoscópica con injerto de cartílago costal 
corticoides, reconstrucciones laringotraqueales con injerto de cartílago con o sin instalación de stents supralaríngeos, entre otros.

\section{Estenosis laringotraqueal}

Las estenosis congénitas pueden presentarse de manera aislada, asociadas a otras malformaciones o como parte de un síndrome. Las estenosis adquiridas pueden producirse por diversas causas como trauma laríngeo, cirugías, infecciones o quemaduras, aunque la gran mayoría son secundarias a daño por intubación endotraqueal. Estas lesiones agudas (como granulomas y úlceras) pueden evolucionar a una estenosis laringotraqueal, en especial si no son detectadas y tratadas de manera oportuna ${ }^{25,26}$.

Conceptualmente, se excluirán de esta definición de estenosis las obstrucciones extrínsecas, por colapso, o por tumores y quistes.

\section{a) Supraglotis}

La mayoría son iatrogénicas o por ingestión de cáusticos. Son lesiones infrecuentes, muchas veces complejas y de manejo poco estandarizado ${ }^{27}$.

\section{b) Glotis}

Las lesiones congénitas incluyen las membranas (webs) y la atresia laríngea. A veces se asocian a síndromes como el velocardiofacial. Cohen clasifica las membranas en 4 grados, de complejidad creciente ${ }^{28}$. El tratamiento puede considerar observación, división endoscópica, reconstrucción laringotraqueal con injerto de cartílago, resección cricotraqueal parcial extendida y/o traqueostomía.

Las lesiones adquiridas corresponden a cicatrices o sinequias, que pueden ubicarse hacia anterior, similar a una membrana, o hacia posterior, generando inmobilidad cordal. La estenosis glótica posterior es habitualmente secundaria a lesiones por intubación. Se clasifica según Bogdasarian en 4 grados según el tipo de compromiso interaritenoideo y de la articulación cricoaritenoidea $^{16}$. Muchas veces coexiste con estenosis subglótica, confiriéndole una mayor complejidad.

\section{c) Subglotis}

La subglotis es una zona anatómica de especial relevancia, ya que es el único anillo cartilaginoso completo, por lo que es menos distensible. Además, es la zona más estrecha de la vía aérea laringotraqueal en niños pequeños.

Myer-Cotton clasifican las estenosis subglóticas en 4 grados, de acuerdo al porcentaje de obstrucción respecto a una vía aérea normal para la edad del paciente: Grado I, de 0 a 50\%; grado II, de 51 a $70 \%$; grado III, de 71 a $99 \%$; y grado IV, $100 \%{ }^{29}$.

La estenosis subglótica congénita puede ser cartila- ginosa, de tejidos blandos o mixta. Es la tercera causa más frecuente de estridor congénito, con un 10-15\% de los casos. De requerir tratamiento, habitualmente se requieren técnicas quirúrgicas abiertas de expansión y/o resección ${ }^{30}$.

La estenosis subglótica adquirida es una causa relativamente frecuente de estridor post-intubación, producida por cicatrices que estrechan el lumen. Pueden ser membranosas o más gruesas, siendo más difíciles de tratar cuando tienen una extensión mayor a $1 \mathrm{~cm}$ o comprometen también la glotis. La prevención incluye disminuir el riesgo de lesiones agudas por intubación, el diagnóstico precoz y el tratamiento efectivo de dichas lesiones. Una vez establecida la estenosis, el tratamiento específico puede incluir dilatación roma o con balón; aplicación de medicamentos tópicos como corticoides o mitomicina C; sección simple; split cricoideo; reconstrucción laringotraqueal (figura 3 ) o resección cricotraqueal parcial ${ }^{25,30,31}$.

\section{d) Tráquea}

Las estenosis traqueales congénitas pueden presentarse de manera aislada o asociadas a malformaciones vasculares, por ejemplo, en anillos traqueales completos asociados a un sling de la arteria pulmonar. Las alteraciones adquiridas pueden ser consecuencia de intubación, traqueostomía u otras cirugías previas sobre la vía aérea.

El tratamiento puede incluir dilatación endoscópica, resección y anastomosis, expansión con injertos, o traqueoplastía por deslizamiento (slide) ${ }^{32}$.

\section{Hendiduras laringotraqueales (clefts)}

Alteraciones congénitas muy infrecuentes que pueden presentarse de forma aislada o asociada a síndromes. Se caracterizan por un defecto en los tejidos que forman la división entre la vía aérea laringotraqueal y la vía digestiva alta. De acuerdo a Benjamin-Inglis, se cla-

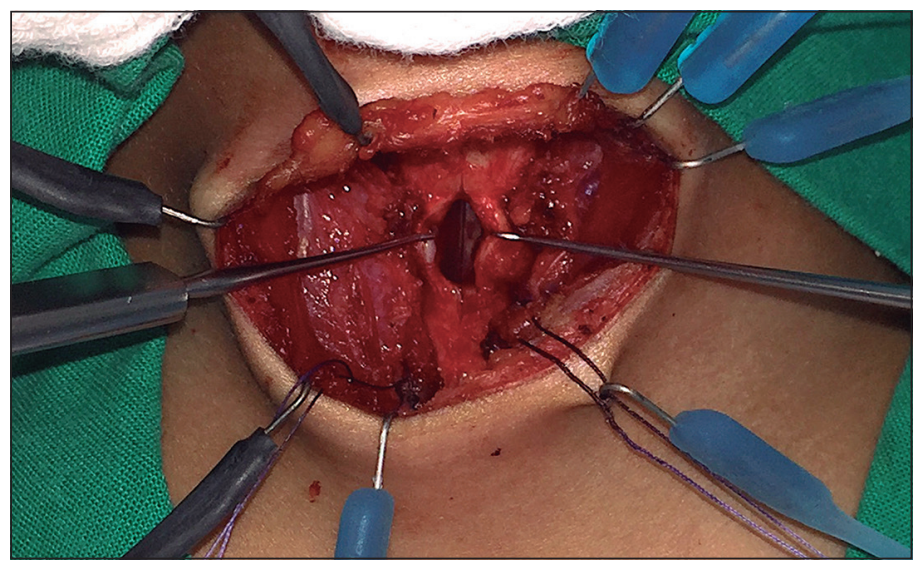

Figura 3. Incisión cricoidea durante una reconstrucción laringotraqueal abierta. 
sifican en 4 grados según su extensión ${ }^{33}$. Clínicamente se manifiestan con una combinación de síntomas respiratorios y deglutorios. El diagnóstico endoscópico requiere la palpación del área interaritenoidea, pues algunos pueden pasar inicialmente desapercibidos. La resolución quirúrgica en pacientes sintomáticos puede realizarse por vía endoscópica o abierta ${ }^{33,34}$.

\section{Hemangioma subglótico}

Tumor vascular benigno que compromete infrecuentemente la laringe. El 50\% de los pacientes tienen otros hemangiomas cutáneos, en especial en la cara. Los síntomas comienzan a los 2-4 meses de edad, aumentando hasta los 10-12 meses, para luego estabilizarse e involucionar progresivamente. Aunque existen varios tratamientos médicos y quirúrgicos descritos, actualmente el propranolol sistémico es el tratamiento de primera línea en la mayoría de los $\operatorname{casos}^{35}$.

\section{Quistes laríngeos}

Incluyen quistes ductales, saculares y laringoceles. Los primeros se deben a obstrucciones de los ductos de glándulas mucosas y pueden ubicarse en distintas regiones como vallécula o subglotis; en esta última suelen asociarse a antecedente de intubación varios meses antes de los síntomas ${ }^{36-38}$. Los quistes saculares y los laringoceles se deben a una dilatación anormal del sáculo laríngeo. El tratamiento puede comprender punción aspirativa, marsupialización o resección completa.

\section{Traqueobroncomalacia}

Debilidad o colapsabilidad de parte o toda la vía aérea traqueobronquial, que puede afectar tanto al anillo cartilaginoso como a la pared posterior membranosa/ muscular. Se considera significativa cuando disminuye más de $50 \%$ del lumen traqueal y puede ser primaria o secundaria, habitualmente asociada a malformaciones esofágicas (atresia esofágica y fístula traqueoesofágica), cardiovasculares o compresiones extrínsecas ${ }^{39}$. También pueden producirse después de una intubación prologada o como secuela de una traqueostomía (colapso supraestomal).

El tratamiento en pacientes sintomáticos suele ser complejo y puede incluir ventilación mecánica no invasiva, traqueostomía, suspensiones directas o indirectas (aortopexia, traqueopexia anterior y/o posterior), stents endoluminales o splints extraluminales.
Los casos primarios suelen resolverse hacia los 2 años de edad.

\section{Movimiento paradójico de cuerdas vocales}

Descoordinación ocasional de las cuerdas vocales, que se cierran en lugar de abrirse en inspiración, manifestándose como estridor inspiratorio intermitente. Está mejor descrito en adolescentes y adultos, pero existen reportes aislados en recién nacidos e infantes ${ }^{40}$. Su etiología es desconocida y el manejo dependerá de la gravedad de los síntomas, muchas veces solo requiriendo observación.

\section{Otras causas de estridor persistente}

Existen otras alteraciones laríngeas capaces de producir estridor, como por ejemplo las secundarias a trauma externo, tumores extra y endoluminales, quemaduras e infecciones crónicas; cuyo diagnóstico y manejo van más allá de los alcances de esta revisión.

\section{Conclusión}

Existen diversas causas congénitas y adquiridas de estridor persistente en la edad pediátrica. Se requiere de una adecuada comprensión de la etiopatogenia y fisiopatología, un acucioso estudio endoscópico y eventualmente imagenológico que permita plantear una estrategia terapéutica individualizada. La alta prevalencia de una segunda patología de la vía aérea y de comorbilidades locales o sistémicas deben ser también consideradas.

El tratamiento puede incluir observación activa, medidas generales y tratamiento del reflujo, ventilación mecánica no invasiva, aplicación local de medicamentos y técnicas quirúrgicas endoscópicas o abiertas orientadas a disminuir el colapso dinámico, expandir la vía aérea o resecar las áreas comprometidas.

Aún así, el manejo de estos pacientes es complejo y requiere un enfoque multidisciplinario, no siempre consiguiendo una resolución completa de los síntomas. En otros casos, una traqueostomía transitoria o permanente puede ser la alternativa más razonable.

\section{Conflicto de intereses}

El autor declara no tener conflicto de intereses. 


\section{Referencias}

1. Monnier P. Clinical Evaluation of Airway Obstruction. En: Monnier P. Pediatric Airway Surgery: Management of Laryngotracheal Stenosis in Infants and Children. Springer-Verlag, Berlin Heidelberg, 2011. pp. 31-44.

2. Nolder AR, Richter GT. The Infant With Noisy Breathing. Curr Treat Options Peds 2015;1:224-33.

3. Alvo A. Evaluación Endoscópica en Otorrinolaringología. En: Alvo A, Breinbauer H. Manual de Otorrinolaringología. Editorial Mediterráneo, 2019. pp. 23-4.

4. Eslamy HK, Newman B. Imaging of the pediatric airway. Paediatr Anaesth 2009;19 Suppl 1:9-23.

5. Su SC Masters IB, Buntain $\mathrm{H}$, et al. A comparison of virtual bronchoscopy versus flexible bronchoscopy in the diagnosis of tracheobronchomalacia in children. Pediatr Pulmonol 2017;52:4806.

6. Boesch RP, Balakrishnan K, Acra S, et al. Structure and Functions of Pediatric Aerodigestive Programs: A Consensus Statement. Pediatrics 2018;141. pii: e20171701.

7. Watters KF. Tracheostomy in Infants and Children. Respir Care 2017;62:799-825.

8. Trachsel D, Hammer J. Indications for tracheostomy in children. Paediatr Respir Rev 2006;7:162-8.

9. Monnier P. Laryngomalacia. En: Monnier P. Pediatric Airway Surgery: Management of Laryngotracheal Stenosis in Infants and Children. Springer-Verlag, Berlin Heidelberg, 2011. pp. 99-106.

10. Gazzaz MJ, El-Hakim H. A case of acquired laryngomalacia in an infant, with endoscopy before and after establishing the diagnosis for the first time. BMJ Case Rep 2015;2015. pii: bcr2015213264.

11. Olney DR, Greinwald JH Jr, Smith RJ, et al. Laryngomalacia and its treatment. Laryngoscope 1999;109:1770-5.

12. Dobbie AM, White DR. Laryngomalacia. Pediatr Clin North Am 2013;60:893-902.

13. Rifai HA, Benoit M, El-Hakim $\mathrm{H}$. Secondary airway lesions in laryngomalacia: a different perspective. Otolaryngol Head Neck Surg 2011;144:268-73.

14. Ayari S, Aubertin G, Girschig H, et al Management of laryngomalacia. Eur
Ann Otorhinolaryngol Head Neck Dis 2013;130:15-21.

15. Richter GT, Thompson DM. The surgical management of laryngomalacia. Otolaryngol Clin North Am 2008;41:83764 , vii.

16. Stephenson KA, Wyatt ME. Glottic stenosis. Semin Pediatr Surg 2016;25:1327.

17. Boudewyns A, Claes J, Van de Heyning P. Clinical practice: an approach to stridor in infants and children. Eur J Pediatr 2010;169:135-41.

18. Rutter MJ. Congenital laryngeal anomalies. Braz J Otorhinolaryngol 2014;80:533-9.

19. Chen EY, Inglis AF Jr. Bilateral vocal cord paralysis in children. Otolaryngol Clin North Am 2008;41:889-901, viii.

20. Howell R, Romeo S, Myer C $4^{\text {th }}$, et al. The lasso technique for endoscopic suture lateralization in bilateral vocal fold immobility. Laryngoscope 2017;127:26047.

21. Rutter MJ, Hart CK, Alarcon A, et al. Endoscopic anterior-posterior cricoid split for pediatric bilateral vocal fold paralysis. Laryngoscope 2018;128:257-63.

22. Thakkar K, Gerber ME. Endoscopic posterior costal cartilage graft placement for acute management of pediatric bilateral vocal fold paralysis without tracheostomy. Int J Pediatr Otorhinolaryngol 2008;72:1555-8.

23. El-Hakim H. Injection of botulinum toxin into external laryngeal muscles in pediatric laryngeal paralysis. Ann Otol Rhinol Laryngol 2008;117:614-20.

24. Daniel SJ, Cardona I. Cricothyroid onabotulinum toxin A injection to avert tracheostomy in bilateral vocal fold paralysis. JAMA Otolaryngol Head Neck Surg 2014;140:867-9.

25. Alvo A, Sedano C. Prevención, diagnóstico y manejo de lesiones laringotraqueales agudas y subagudas posintubación en pacientes pediátricos. Rev Otorrinolaringol Cir Cabeza Cuello 2017;77:91-8.

26. Schweiger C, Manica D, Kuhl G, et al. Post-intubation acute laryngeal injuries in infants and children: A new classification system. Int J Pediatr Otorhinolaryngol 2016;86:177-82.

27. Colliard A, Ishii A, De Sandre C, et al. Decoding supraglottic stenosis. Eur Arch Otorhinolaryngol 2020;277:293-300.
28. de Trey LA, Lambercy K, Monnier P, et al. Management of severe congenital laryngeal webs - a 12 year review. Int J Pediatr Otorhinolaryngol 2016;86:82-6.

29. Myer CM 3rd, O'Connor DM, Cotton RT. Proposed grading system for subglottic stenosis based on endotracheal tube sizes. Ann Otol Rhinol Laryngol 1994;103:31923.

30. Monnier P. Congenital Subglottic Stenosis. En: Monnier P. Pediatric Airway Surgery: Management of Laryngotracheal Stenosis in Infants and Children. Springer-Verlag, Berlin Heidelberg, 2011. pp. 119-24.

31. Alvo A, Villarroel G, Castro S, Chávez C, Sedano C. Dilatación precoz de estenosis subglótica adquirida posintubación utilizando tubos endotraqueales. Rev Otorrinolaringol Cir Cabeza Cuello 2019;79:271-8.

32. Ho AS, Koltai PJ. Pediatric tracheal stenosis. Otolaryngol Clin North Am 2008;41:999-1021, x.

33. Landry AM, Rutter MJ. Airway Anomalies. Clin Perinatol 2018;45:597607.

34. Coppess S, Padia R, Horn D, et al. Standardizing Laryngeal Cleft Evaluations: Reliability of the Interarytenoid Assessment Protocol. Otolaryngol Head Neck Surg 2019;160:533-9.

35. Darrow DH. Management of Infantile Hemangiomas of the Airway. Otolaryngol Clin North Am 2018;51:133-46.

36. Zawadzka-Glos L, Frackiewicz M, Brzewski M, et al. Difficulties in diagnosis of laryngeal cysts in children. Int J Pediatr Otorhinolaryngol 2009;73:1729-31.

37. Cunha MS, Janeiro P, Fernandes R, et al. Congenital laryngomucocoele: a rare cause for CHAOS. BMJ Case Rep 2009;2009. pii: bcr07.2008.0595.

38. Lim J, Hellier W, Harcourt J, et al. Subglottic cysts: the Great Ormond Street experience. Int J Pediatr Otorhinolaryngol 2003;67:461-5.

39. Alvo A, Sedano C, van der Meer G. Traqueomalacia pediátrica. Rev Otorrinolaringol Cir Cabeza Cuello 2019;79:347-56.

40. O'Connell Ferster AP, Shokri T, Carr M. Diagnosis and treatment of paradoxical vocal fold motion in infants. Int J Pediatr Otorhinolaryngol 2018;107:6-9. 